\title{
NONISOMORPHIC ALGEBRAIC MODELS OF A SMOOTH MANIFOLD WITH GROUP ACTION
}

\author{
KARL HEINZ DOVERMANN, MIKIYA MASUDA, AND DONG YOUP SUH
}

(Communicated by James West)

\begin{abstract}
Let $G$ be a finite group and $M$ a closed smooth $G$ manifold. If $M$ has any equivariant algebraic model, then we show that it has uncountable many birationally inequivalent such models. This generalizes a non-equivariant result of Bochnak and Kucharz.
\end{abstract}

\section{INTRODUCTION}

In this note we show

Theorem A. Let $G$ be a finite group and $X$ a compact non-singular real algebraic $G$ variety of dimension at least 1 . Then there exists an uncountable family $\left\{X_{\alpha}\right\}_{\alpha \in \Lambda}$ of non-singular real algebraic $G$ varieties such that

(1) considered as smooth $G$ manifolds, $X_{\alpha}$ is equivariantly diffeomorphic to $X$ and

(2) $X_{\alpha}$ is not birationally equivalent to $X_{\beta}$ for $\alpha \neq \beta$.

In other words, there are uncountably many different algebraic $G$ models for the smooth $G$ manifold underlying a compact non-singular real algebraic $G$ variety. Our Theorem $A$ is the equivariant version of the theorem proved by Bochnak and Kucharz in [BK]. We left out certain technical parts of their statement as we do not want to concern ourselves with specific embedding questions. Theorem A does not address the problem of whether a given closed smooth $G$ manifold has an algebraic model, i.e., whether it is equivariantly diffeomorphic to a non-singular real algebraic $G$ variety. In this case we say that the manifold is algebraically realized. Unlike in the setting without group action, where Tognoli proved that every closed smooth manifold is algebraically realized [T], we have only the following partial result.

Theorem (see [DM], [DMS], [DKS], and [KM]). Let $G$ be a compact Lie group. $A$ closed smooth $G$ manifold is algebraically realized if one of the following assumptions holds.

(1) $G$ is the product of a group of odd order and a 2-torus.

Received by the editors April 17, 1993.

1991 Mathematics Subject Classification. Primary 14P05, 57S17.

The research of K. H. Dovermann at MSRI is supported in part by NSF grant \# DMS9022140.

D. Y. Suh is partially supported by the Korea Science and Engineering Foundation. 
(2) The action of $G$ on the manifold is semifree.

(3) The manifold is of dimension two.

Under the assumptions of this theorem, Theorem A may be turned into a result about the algebraic structures on a closed smooth $G$ manifold.

Our proof of Theorem A follows the one of Bochnak and Kucharz closely. Needed is an algebraic approximation result, and here we replace a reference to $[\mathrm{AK}]$ by an equivariant result of [DMS]; see Theorem 2.1. To make the paper accessible to the non-expert, we summarize two essential arguments from algebraic geometry in Propositions 2.2 and 2.3, which we then prove in Section 3. We would like to thank Professor W. Fulton for a careful reading of the first draft of this note and Professors S. H. Bae and A. Corti for some useful discussions on elliptic curves and abelian varieties.

\section{Proof of Theorem A}

Throughout, $G$ denotes a finite group. We recall some basic definitions. Let $\Omega$ be an orthogonal representation of $G$. Here we think of an orthogonal representation as an underlying Euclidean space $\mathbb{R}^{n}$ together with an action of $G$ via orthogonal maps. A real algebraic $G$ variety is the set of common zeros of a finite set of polynomials $p_{1}, \ldots, p_{m}: \Omega \rightarrow \mathbb{R}, V=\left\{x \in \Omega \mid p_{1}(x)=\right.$ $\left.\cdots=p_{m}(x)=0\right\}$, which is invariant under the action of $G$. See [DM] for an equivalent, more algebraically minded definition.

Let $f_{i}: M_{i} \rightarrow Y$ be equivariant maps whose domain is a closed smooth $G$ manifold, $i=1,2$. They are said to be equivariantly cobordant if there exists a compact smooth $G$ manifold $W$ and an equivariant map $F: W \rightarrow Y$, such that the boundary of $W$ is the disjoint union of $M_{1}$ and $M_{2}$ and $F$ restricts to $f_{i}$ on $M_{i}$. Suppose that $Y$ is a real algebraic $G$ variety. We say that $f: M \rightarrow Y$ is algebraically realized if there exists a non-singular real algebraic $G$ variety $X$, an equivariant entire rational map $\mu: X \rightarrow Y$, and an equivariant diffeomorphism $\phi: M \rightarrow X$, such that $\mu \circ \phi$ is equivariantly homotopic to $f$. We say that $(M, f)$ can be approximated algebraically if there are algebraic realizations $(X, \mu, \phi)$ such that $\mu \circ \phi$ is arbitrarily close to $f$ in the $C^{1}$ topology. An equivariant bordism class is said to be algebraically realized if it has a representative $\mu: X \rightarrow Y$ where $X$ is a non-singular real algebraic $G$ variety and $\mu$ is an equivariant entire rational map. In [DMS, Theorem $\mathrm{C}$ and its addendum in Section 7] we showed

Theorem 2.1. Let $G$ be a compact Lie group. An equivariant map from a closed smooth $G$ manifold to a non-singular real algebraic $G$ variety can be approximated algebraically if and only if its equivariant bordism class is algebraically realized.

In Section 3, we define 'isogeny' and prove the following two propositions. The first proposition is used to construct an uncountable family of algebraic models, the second one to distinguish them.

Proposition 2.2. There exists an uncountable family $\left\{E_{\alpha}\right\}_{\alpha \in \Lambda}$ of non-singular complex cubic curves in $\mathbb{C} P^{2}$ such that each $E_{\alpha}$ is defined over $\mathbb{R}$, the real cubic curve $D_{\alpha}=E_{\alpha} \cap \mathbb{R} P^{2}$ is connected, and $E_{\alpha}$ is not isogenous to $E_{\beta}$ for $\alpha \neq \beta$. 
Proposition 2.3. Suppose for every $\alpha$ in an index set $\Lambda$ we are given an elliptic curve $E_{\alpha}$, an irreducible projective variety $V_{\alpha}$, and a non-constant rational map $F_{\alpha}: V_{\alpha} \rightarrow E_{\alpha}$. Assume that $E_{\alpha}$ is not isogenous to $E_{\beta}$ if $\alpha \neq \beta$. Then only a finite number of the $V_{\alpha}, \alpha \in \Lambda$, are in any given birational equivalence class.

The need for the analysis which we are about to describe arises because in the proof of Theorem A we are first dealing with affine varieties when we apply Theorem 2.1, and later we are treating them as projective varieties when we apply Proposition 2.3. As described, $D_{\alpha} \subset \mathbb{R} P^{2}$ and $E_{\alpha} \subset \mathbb{C} P^{2}$. One may check that $E_{\alpha}$ is the Zariski closure of $D_{\alpha}$ in $\mathbb{C} P^{2}$. In the language of Whitney [W], we call $E_{\alpha}$ the complexification of $D_{\alpha}$. We identify $\mathbb{R} P^{2}$ with the Grassmannian $G_{1}\left(\mathbb{R}^{3}\right) \subset \mathbb{R}^{9}$, and embed it into $\mathbb{R} P^{9}$. Call the composition of the embeddings $l: \mathbb{R} P^{2} \rightarrow \mathbb{R} P^{9}$, and we arrange it so that $l$ is a regular embedding. (We will be more explicit in the next paragraph, giving formal expressions for these embeddings such that our statements may be checked.) By the same formal expression we get a regular embedding $l: \mathbb{C} P^{2} \rightarrow \mathbb{C} P^{9}$. Observe that $l\left(\mathbb{C} P^{2}\right)$ is the complexification of $l\left(\mathbb{R} P^{2}\right)$ in $\mathbb{C} P^{9}$. The Zariski closure of $l\left(D_{\alpha}\right)$ in $\mathbb{C} P^{9}$ is then the Zariski closure of $l\left(D_{\alpha}\right)$ in $l\left(\mathbb{C} P^{2}\right)$, so the complexification of $l\left(D_{\alpha}\right)$ in $\mathbb{C} P^{9}$ is $l\left(E_{\alpha}\right)$. Keeping this analysis in mind, we consider $D_{\alpha}$ as a curve in $\mathbb{R} P^{2}$ and in $\mathbb{R} P^{9}$, and $E_{\alpha}$ as a curve in $\mathbb{C} P^{2}$ and in $\mathbb{C} P^{9}$. With both interpretations, $E_{\alpha}$ is the Zariski closure of $D_{\alpha}$.

The identification of a point $[X, Y, Z] \in \mathbb{R} P^{2}$ in homogeneous coordinates with an element in the Grassmannian

$$
G_{1}\left(\mathbb{R}^{3}\right)=\left\{L \in \mathfrak{M}_{3 \times 3} \mid L^{2}=L, L^{t}=L, \operatorname{tr} L=1\right\}
$$

(here $\mathfrak{M}_{3 \times 3}$ stands for the $3 \times 3$ matrices with real coefficients) assigns to a point in $\mathbb{R} P^{2}$ the orthogonal projection onto the line through this point, i.e.,

$$
[X, Y, Z] \mapsto \frac{1}{X^{2}+Y^{2}+Z^{2}}\left(\begin{array}{ccc}
X X & Y X & Z X \\
X Y & Y Y & Z Y \\
X Z & Y Z & Z Z
\end{array}\right) .
$$

We identify $\mathfrak{M}_{3 \times 3}$ with $\mathbb{R}^{9}$ and embed it into $\mathbb{R} P^{9}$ mapping

$$
\begin{aligned}
\left(\begin{array}{lll}
u_{0} & w_{2} & w_{1} \\
v_{2} & u_{1} & w_{0} \\
v_{1} & v_{0} & u_{2}
\end{array}\right) & \mapsto\left(u_{0}, u_{1}, u_{2}, v_{0}, v_{1}, v_{2}, w_{0}, w_{1}, w_{2}\right) \\
& \mapsto\left[u_{0}, u_{1}, u_{2}, v_{0}, v_{1}, v_{2}, w_{0}, w_{1}, w_{2}, 1\right] .
\end{aligned}
$$

A polynomial expression for the composition of these two maps is given by

$$
[X, Y, Z] \mapsto\left[X X, Y Y, Z Z, Y Z, X Z, X Y, Z Y, Z X, Y X, X^{2}+Y^{2}+Z^{2}\right] \text {. }
$$

This is the explicit description of the map $l: \mathbb{R} P^{2} \rightarrow \mathbb{R} P^{9}$ considered in the previous paragraph. Using coordinates $\left[u_{0}, u_{1}, u_{2}, v_{0}, v_{1}, v_{2}, w_{0}, w_{1}, w_{2}, z\right]$ to describe points in $\mathbb{R} P^{9}$ one may see that $l\left(\mathbb{R} P^{2}\right)$ is described by the following equations as a subvariety in $\mathbb{R} P^{9}$ :

$$
\begin{aligned}
& v_{0}^{2}-u_{1} u_{2}=0, \quad v_{0}=w_{0}, \\
& v_{1}^{2}-u_{0} u_{2}=0, \quad v_{1}=w_{1}, \\
& v_{2}^{2}-u_{0} u_{1}=0, \quad v_{2}=w_{2}, \\
& u_{0}^{2}+u_{1}^{2}+u_{2}^{2}+v_{0}^{2}+v_{1}^{2}+v_{2}^{2}+w_{0}^{2}+w_{1}^{2}+w_{2}^{2}=z^{2} .
\end{aligned}
$$


The polynomial expression in $(*)$ defines an embedding $l: \mathbb{C} P^{2} \rightarrow \mathbb{C} P^{9}$, and $\imath\left(\mathbb{C} P^{2}\right)$ is also described as a subvariety of $\mathbb{C} P^{9}$ by the equations which we just gave. With these definitions one may check the claims in the previous paragraph.

Let $D$ be a set, then we consider the set $\operatorname{Map}(G, D)$ of all maps from $G$ to $D$. An action

$$
\theta: G \times \operatorname{Map}(G, D) \rightarrow \operatorname{Map}(G, D)
$$

of $G$ is defined by setting $\theta(g, f)(h)=f(h g)$. If $D$ is a non-singular real algebraic variety, then $\operatorname{Map}(G, D)$ is a non-singular real algebraic $G$ variety, in fact, it is the $|G|$-fold cartesian product of $D$, and $G$ acts by permuting coordinates. Let $x$ be a fixed point in $\operatorname{Map}(G, D)$ and $d=\operatorname{dim}_{\mathbb{R}} D$. The tangent representation $T_{x} \operatorname{Map}(G, D)$ at $x$ is the $d$-fold multiple of the regular representation $\operatorname{Map}(G, \mathbb{R})=\mathbb{R}[G]$.

Proof of Theorem A. In our first step we construct an uncountable family $\left\{X_{\alpha}\right\}_{\alpha \in \Lambda}$ of equivariant algebraic models of $X$. In the second step we choose an uncountable subset of birationally inequivalent models.

Let $\left\{D_{\alpha}\right\}_{\alpha \in \Lambda}$ be an uncountable family of real projective curves as in Proposition 2.2. Let $\mathbb{D}_{\alpha}$ be the $k$-fold cartesian product of $\operatorname{Map}\left(G, D_{\alpha}\right)$. We determine the value for $k$ by the following construction. Note that $D_{\alpha}$ is nonsingular and so is $\mathbb{D}_{\alpha}$. By definition, $X$ is a subset of some representation of $G$, so we may suppose that it is contained in any sufficiently large multiple $k \mathbb{R}[G]$ of the regular representation of $G$. Identify $k \mathbb{R}[G]$ with the equivariant neighbourhood of a fixed point $y$ in $\mathbb{D}_{\alpha}$; then we find a smooth equivariant embedding $f: X \rightarrow \mathbb{D}_{\alpha}$. Define $\mu: X \rightarrow \mathbb{D}_{\alpha}$ by $\mu(x)=y$ for all $x \in X$, and observe that $(X, \mu)$ is an algebraic representative of $(X, f)$. Theorem 2.1 provides us with equivariant algebraic approximations $\left(X_{\alpha}, f_{\alpha}\right)$ of $(X, f)$. Approximating $(X, f)$ closely enough, we may suppose that $f_{\alpha}$ is nonconstant on each component of $X_{\alpha}$. This provides us with an uncountable family $\left\{X_{\alpha}\right\}_{\alpha \in \Lambda}$ of equivariant algebraic models of $X$, completing the first step.

From now on the argument is non-equivariant. Composition with projection on an appropriate factor of $\mathbb{D}_{\alpha}$ provides us with an entire rational function $f_{\alpha}^{\prime}: X_{\alpha} \rightarrow D_{\alpha}$ which is nonconstant on each component of $X_{\alpha}$. To fix notation, we say that $X_{\alpha}$ is realized as a zero set of polynomials in $\mathbb{R}^{n(\alpha)}$ and, in the sense of the discussion from above, $D_{\alpha}$ is realized as an affine variety in $\mathbb{R}^{9}$. Then, by definition, we have an entire rational extension $\widetilde{f}_{\alpha}^{\prime}: \mathbb{R}^{n(\alpha)} \rightarrow \mathbb{R}^{9}$ of $f_{\alpha}^{\prime}$. As for $n=9$, we choose an embedding $j: \mathbb{R}^{n} \rightarrow \mathbb{R} P^{n}$ mapping $\left(x_{1}, \ldots, x_{n}\right)$ to $\left[x_{1}, \ldots, x_{n}, 1\right]$. Then $\widetilde{f}_{\alpha}^{\prime}$ induces a rational mapping $\bar{f}_{\alpha}: \mathbb{R} P^{n(\alpha)} \rightarrow \mathbb{R} P^{9}$ by setting $\bar{f}_{\alpha}\left(\left[x_{1}, \ldots, x_{n}, z\right]\right)=\left[\tilde{f}_{\alpha}^{\prime}\left(x_{1} / z, \ldots, x_{n} / z\right), 1\right]$. This map restricts to a rational map of real projective varieties, $h_{\alpha}: j\left(X_{\alpha}\right) \rightarrow D_{\alpha} \subset \mathbb{R} P^{9}$ which is nonconstant on each component of $j\left(X_{\alpha}\right)$. Let $W_{\alpha} \subset \mathbb{C} P^{n(\alpha)}$ be the complexification of $j\left(X_{\alpha}\right)$, i.e., $W_{\alpha}$ is the Zariski closure of $j\left(X_{\alpha}\right)$ in $\mathbb{C} P^{n(\alpha)}$. Remember that $E_{\alpha}$ is the complexification of $D_{\alpha}$, and observe that $h_{\alpha}$ induces a rational map $F_{\alpha}: W_{\alpha} \rightarrow E_{\alpha}$ which is nonconstant on each component of $W_{\alpha}$. Because we assumed that $E_{\alpha}$ is not isogenous to $E_{\beta}$ if $\alpha \neq \beta$, it follows from Proposition 2.3 that only a finite number of the irreducible components of the $W_{\alpha}$, hence of the $W_{\alpha}$ themselves, are in any given birational equivalence class. We may then choose an uncountable subset $\Lambda^{\prime}$ of $\Lambda$ such that $W_{\alpha}$ is not bira- 
tionally equivalent to $W_{\beta}$ if $\alpha, \beta \in \Lambda^{\prime}$ and $\alpha \neq \beta$. If $X_{\alpha}$ is birationally equivalent to $X_{\beta}$, then $W_{\alpha}$ is birationally equivalent to $W_{\beta}$. This implies that $X_{\alpha}$ is not birationally equivalent to $X_{\beta}$ if $\alpha, \beta \in \Lambda^{\prime}$ and $\alpha \neq \beta$. This completes the proof.

\section{REVIEW OF RESULTS FROM ALGEBRAIC GEOMETRY}

Consider a plane cubic curve $e$ over $\mathbb{C}$ defined by the Weierstrass equation

$$
y^{2}=x^{3}+A x+B \text {. }
$$

Its discriminant is defined as $\Delta(e)=-16\left(4 A^{3}+27 B^{2}\right)$ and its $j$-invariant as $j(e)=1728(4 A)^{3} / \Delta(e)$ (compare [S, p. 50]). The plane curve $e$ defines a projective curve

$$
E=\left\{[X, Y, Z] \in \mathbb{C} P^{2} \mid Y^{2} Z=X^{3}+A X Z^{2}+B Z^{3}\right\} .
$$

They are related by the coordinate transformation $x=X / Z$ and $y=Y / Z$, and $E$ is obtained from $e$ by adding the distinguished point $[0,1,0]$. We also use the notation $\Delta(E)$ and $j(E)$ with the obvious meaning.

Proposition 3.1. Let $A, B, e$, and $E$ be as above.

(1) If $\Delta(e) \neq 0$, then the plane curve $e$ and the projective curve $E$ are non-singular and $E$ is elliptic.

(2) If $A$ and $B$ are real and $\Delta(e)<0$, then the real projective curve $D=$ $E \cap \mathbb{R} P^{2}$ is connected and non-singular.

Proof. A Weierstrass plane cubic curve is non-singular if and only if its discriminant is non-zero [S, p. 50], i.e., if it has three distinct roots. The projective curve $E$ will then be non-singular at $[0,1,0]$, so that it is smooth. This implies that $E$ is elliptic [S, p. 63].

If $A$ and $B$ are real and $\Delta(e)<0$, then $x^{3}+A x+B=0$ has exactly one real solution. This implies that $D=\left\{[X, Y, Z] \in \mathbb{R} P^{2} \mid Y^{2} Z=X^{3}+A X Z^{2}+\right.$ $\left.B Z^{3}\right\}$ is connected. It follows from an elementary argument using the CauchyRiemann equations that $D$ is non-singular if $E$ is non-singular because both curves are defined by the same equation with real coefficients.

Two abelian varieties $E$ and $E^{\prime}$ are called isogenous if there exists a surjective homomorphism $\mu: E \rightarrow E^{\prime}$ with finite kernel. Isogeny is an equivalence relation. Elliptic curves are examples of simple abelian varieties, i.e., varieties which, by definition, have no non-trivial proper abelian subvarieties. In fact, any non-constant rational map between elliptic curves which preserves the distinguished point is an isogeny. (Rationality implies that the map is regular [S, p. 23], regularity at every point means by definition that the map is a morphism [S, p. 16], and by a well-known theorem every non-trivial homomorphism between elliptic curves is an isogeny. In [S, p. 70] this is used as the definition for the term isogeny.) For any elliptic curve $E$ the set of all elliptic curves isogenous to $E$ is countable [H, p. 338].

Proof of Proposition 2.2. Set $A=1$ and let $B$ be any positive real number. Then we obtain a projective curve $E_{B}=\left\{[X, Y, Z] \in \mathbb{C} P^{2} \mid Y^{2} Z=X^{3}+\right.$ $\left.X Z^{2}+B Z^{3}\right\}$. Apparently, $\Delta\left(E_{B}\right)<0$, so that the curve is non-singular (see $3.1(1)$ ), and $D_{B}=E_{B} \cap \mathbb{R} P^{2}$ is connected and non-singular (see 3.1(2)). If 
$B \neq B^{\prime}$, then $j\left(E_{B}\right) \neq j\left(E_{B^{\prime}}\right)$. The $j$-invariant determines an elliptic curve up to isomorphism [S, p. 50], and it follows that $E_{B}$ and $E_{B^{\prime}}$ are not isomorphic. As there are uncountably many values for $B$, and only a countable number of the curves are in the same isogeny class, we may pick one curve from each class and obtain the desired uncountable family $\left\{E_{\alpha}\right\}_{\alpha \in \Lambda}$ of cubic curves.

The material on abelian varieties used in the following discussion can be found in [L], mostly in the second chapter where the author discusses the Albanese. Taking the Albanese assigns to a projective variety $V$ an abelian variety $\mathrm{Alb}(V)$. The Albanese has the following universal mapping property. There exists a rational map $\psi: V \rightarrow \operatorname{Alb}(V)$ such that for every rational map $g: V \rightarrow B$ to an abelian variety $B$ there exists a homomorphism $g_{*}: \operatorname{Alb}(V) \rightarrow B$ and a constant $c \in B$ such that $g=g_{*} \psi+c$.

Proof of Proposition 2.3. Let $\bar{V}_{\alpha}$ be the desingularization of $V_{\alpha}$ and $g_{\alpha}: \bar{V}_{\alpha} \rightarrow$ $E_{\alpha}$ the composition of the projection $\bar{V}_{\alpha} \rightarrow V_{\alpha}$ and $F_{\alpha}: V_{\alpha} \rightarrow E_{\alpha}$. Let $\psi_{\alpha}: \bar{V}_{\alpha} \rightarrow \operatorname{Alb}\left(\bar{V}_{\alpha}\right)$ be the universal map discussed before. The universal mapping property provides us with a homomorphism $\left(g_{\alpha}\right)_{*}: \operatorname{Alb}\left(\bar{V}_{\alpha}\right) \rightarrow E_{\alpha}$ and a constant $c_{\alpha} \in E_{\alpha}$ such that $g_{\alpha}=\left(g_{\alpha}\right)_{*} \psi_{\alpha}+c_{\alpha}$. Because $F_{\alpha}$ and $g_{\alpha}$ are non-constant, $\left(g_{\alpha}\right)_{*}$ is non-constant as well.

Because it is an abelian variety, $\operatorname{Alb}\left(\bar{V}_{\alpha}\right)$ is isogenous to a product $A_{\alpha 1} \times$ $\cdots \times A_{\alpha k(\alpha)}$ of simple abelian varieties which are uniquely determined up to order and isogeny [L, p. 30]. The restriction of the composition of this isogeny with $\left(g_{\alpha}\right)_{*}$ to an appropriate factor in this product provides us with a nontrivial homomorphism $h_{\alpha}: A_{\alpha j} \rightarrow E_{\alpha}$, which must be an isogeny because $A_{\alpha j}$ is simple.

A birational equivalence $V_{\alpha} \rightarrow V_{\beta}$ induces a birational equivalence $\bar{V}_{\alpha} \rightarrow \bar{V}_{\beta}$ of the desingularizations, which in turn induces an isomorphism $\operatorname{Alb}\left(\bar{V}_{\alpha}\right) \rightarrow$ $\operatorname{Alb}\left(\bar{V}_{\beta}\right)$. In particular, $E_{\beta}$ is isogenous to one of the simple abelian varieties $A_{\alpha 1}, \cdots, A_{\alpha k(\alpha)}$. Because we assumed that no two of these elliptic curves are isogenous, this is possible for only finitely many $\beta \in \Lambda$. For this reason, $V_{\beta}$ can be birationally equivalent to $V_{\alpha}$ for only finitely many $\beta \in \Lambda$. This was our claim.

\section{REFERENCES}

[AK] S. Akbulut and H. King, The topology of real algebraic sets with isolated singularities, Ann. of Math. (2) 113 (1981), 425-446.

[BK] J. Bochnak and W. Kucharz, Nonisomorphic algebraic models of a smooth manifold, Math. Ann. 290 (1991), 1-2.

[DKS] K. H. Dovermann, F. Knop, and D. Y. Suh, Topological invariants of real algebraic actions, Topology Appl. 40 (1991), 171-188.

[DM] K. H. Dovermann and M. Masuda, Algebraic realization of manifolds with group actions, Adv. Math. (to appear).

[DMS] K. H. Dovermann, M. Masuda, and D. Y. Suh, Algebraic realization of equivariant vector bundles, J. Reine Angew. Math. 448 (1994).

[H] R. Hartshorne, Algebraic geometry, Graduate Texts in Math., vol. 52, Springer-Verlag, Berlin, Heidelberg, and New York, 1977.

[KM] S. S. Kim and M. Masuda, Topological characterizations of non-singular real algebraic $G$-surfaces, Topology Appl. (to appear).

[L] S. Lang, Abelian varieties, Interscience, New York, 1953. 
[S] J. H. Silvermann, The arithmetic of elliptic curves, Graduate Texts in Math., vol. 106, Springer-Verlag, Berlin, Heidelberg, and New York, 1986.

[T] A. Tognoli, Su una Congettura di Nash, Ann. Scuola Norm. Sup. Pisa Cl. Sci. (4) 27 (1973), 167-185.

[W] H. Whitney, Elementary structures of real algebraic varieties, Ann. of Math. (2) 66 (1957), 545-556.

Department of Mathematics, University of Hawail, Honolulu, Hawail 96822-2273

E-mail address: dover@uhunix.bitnet

Department of Mathematics, Osaka City University, Sugimoto, Osaka 558, Japan

E-mail address: masuda@tansei.cc.u-tokyo.ac.jp

Department of Mathematics, Korea Advanced Institute of Science \& Technology, 373-1 Kusong-Dong, Yusong-Gu, TAEJon 305-701, Korea

E-mail address: dysuh@ math1.kaist.ac.kr 